\title{
ON SET-HARMONIOUS LABELING OF SOME GRAPHS
}

\author{
Seema Mehra ${ }^{1}$, Rani Puneet ${ }^{2} \S$ \\ ${ }^{1,2}$ Department of Mathematics \\ Maharshi Dayanand University \\ Rohtak, Haryana, INDIA
}

\begin{abstract}
The aim of this paper is to introduce a new concept of set-harmonious labeling which is an extension of harmonious labeling and also characterize some graph classes with this new labeling.
\end{abstract}

AMS Subject Classification: $47 \mathrm{H} 10,54 \mathrm{H} 25$

Key Words: set-labeling, harmonious labeling, set-harmonious labeling

\section{Introduction}

Graph labeling of graphs is the process of assigning the labels (numbers) to the elements of the graph under some conditions. Due to its many practical applications in X-ray crystallography, computer science, coding theory and many other branches, it becomes the active area of research. All terms and definitions are not mentioned in this paper, we refer to [10] and [5] for more details. The concept of Graph Labeling as $\beta$-valuation was introduced by Rosa [6] in 1967. Subsequently, in 1972, Golomb [3] called it graceful-labeling. Due to phasing the problem in error correcting codes and channel assignments, Graham and Sloane [4] introduced the concept of harmonious labeling by producing the modular version over the graceful labeling. In 1983, Acharya [1] introduced the concept of set-labeling. After words, many authors Acharya et al. [2], Sudev and Germina

\footnotetext{
Received: $\quad$ July 6, 2016

Revised: October 23, 2016

Published: December 16, 2016

$\S_{\text {Correspondence author }}$
}

(c) 2016 Academic Publications, Ltd. url: www.acadpubl.eu 
[8], Thomas and Mathew [9] obtained results on set-labeling.

In this paper we introduce a new concept of set-harmonious labeling which is an extension of harmonious labeling and also characterize some graph classes with this new labeling.

\section{Preliminaries}

Definition 2.1 (Graceful Labeling [6]). Let $G(V, E)$ be a graph with $p$, $q$ number of vertices and edges respectively. If $f$ is any injective function in $G$ defined as $f: V(G) \rightarrow\{0,1,2, \ldots, q\}$ produced a bijective function $f^{*}$ : $E(G) \rightarrow\{0,1,2, \ldots, q\}$ defined as $f^{*}(u v)=|f(u)-f(v)|$ then $f$ is called graceful labeling.

Definition 2.2 (Harmonious Labeling [4]). A graph $G(V, E)$ with $p$ vertices and $q$ edges is said to be harmonious if an injective function $f: V(G) \rightarrow Z_{q}$ produced a bijective function $f^{*}: E(G) \rightarrow Z_{q}$ defined as $f^{*}(u v)=(f(u)+$ $f(v))(\bmod q)$ and $f$ is called harmonious labeling.

Definition 2.3 (Set-Labeling [1]). A set-labeling of a graph $G(V, E)$ is the assignment of unique elements of the power set $2^{X}$ of any non-empty set $X$ to the vertices or edges or both in the graph.

Definition 2.4 (Sumset [7]). The sumset of two non-empty sets $A$ and $B$ denoted by $A+B$ and it have the elements as $A+B=\{c ; c=a+b, a \in A, b \in$ $B$ \}. If any of the sets $A$ and $B$ is countable infinite then their sum sets $\mathrm{A}+\mathrm{B}$ is also countable infinite. Here, we assume $A$ and $B$ as non-empty finite sets.

Definition 2.5 (Cardinality [7]). The number of elements in any set $A$ is called its cardinality. It is denoted as $|A|$.

\section{Main Results}

Motivated by the concept of set-labeling and harmonious labeling of graphs, we introduce set-harmonious labeling. We also study the properties of setharmonious labeling on certain classes of graphs like path, star, double star etc.

Definition 3.1. Let $G(V, E)$ be any graph with non-empty finite set $X$ of non-negative integers. Let $f: V(G) \rightarrow 2^{X}$ be an injective function and it produced a bijective mapping $f^{*}: E(G) \rightarrow 2^{X}-\phi$ defined as $f^{*}(u v)=$ 
$f(u) \widehat{+} f(v)$ where $|X|$ denotes the cardinality of set $X$ and $\hat{+}$ is defined as $f(u) \widehat{+} f(v)=\{r ; a+b=r(\bmod |X|), a \in f(u), b \in f(v)\}$, then $f$ is called set-harmonious labeling and the graph $G$ is called set-harmonious graph.

Major characteristics of set-harmonious graphs are described as under:

Proposition 3.2. If $f: V(G) \rightarrow 2^{X}$ is a set-harmonious labeling on a graph $G$ with $p=q+1$ where $p, q$ are number of vertices and edges respectively. Then, the ground set $X$ must contain 0 as an element.

Proof. Suppose $X$ does not contain 0 as an element. Let $A$ and $B$ are the set-labels of vertices $u$ and $v$ respectively. After taking the sumset of $A$ and $B$ over the modulo $(|X|)$, the label for the edge $u v$ contain 0 . But 0 does not belongs to $X$.

So, when a graph admits a set-harmonious labeling then $X$ must have 0 as an element.

Proposition 3.3. Let $G(V, E)$ be a graph with $p=q+1$. If $f: V(G) \rightarrow$ $2^{X}$ is a set-harmonious labeling on the graph $G$ then, $X$ must be assigned to the pendant vertex in $G$.

Proof. Suppose $X$ is not assigned to the pendant vertex in a graph $G$ with $p=q+1$. So, it is assigned to a label of degree at least 2 . Then, $X$ must be the set-label of at least two edges under modulo $(|X|)$ sumset operation. So, the function along the edges does not become bijective. Hence, it is contradiction to the assumed condition.

Proposition 3.4. If $f: V(G) \rightarrow 2^{X}$ is a set-harmonious labeling on a graph $G(V, E)$ with finite set of non-negative integers $X$ then, $\{0\}$ is either assigned to the vertex which is adjacent to the vertex of set-label $\phi$ or if an element $X_{r}$ in $X$ is the set-label of the vertex $V$ of $G$ then its adjacent vertex have set-label $X_{S}$ if $X_{r}+X_{S}=|X|$, where $|X|$ is the cardinality of the set $X$.

Proof. Let $G$ be a graph with set-harmonious labeling $f$ then, all the sub sets of $X$ except $\phi$ should be the set-label of all edges in $G$. So, $\{0\}$ must be the set-label of one of the edge among them. After applying the sumset modulo $(|X|)$ among the vertices, the edge have set-label $\{0\}$ if and only if either $\{0\}$ is adjacent to $\phi$ or when non-zero any element $X_{r}$ is added into another element $X_{S}$ of the ground set $X$ such that $X_{r}+X_{S}=|X|$. After taking the modulo $(|X|)$, we get $\{0\}$. 
Proposition 3.5. Let $X_{r}$ be any non-empty, non zero subset of $X$ and $f: V(G) \rightarrow 2^{X}$ is a set-harmonious labeling on a graph $G$ then, $\{0\}$ and $\phi$ cannot be the assigned to adjacent vertices of $X_{r}$.

Proof. Suppose $v_{1}$ and $v_{2}$ are two non-adjacent vertices in a graph $G$ and $v_{r}$ is a vertex which is adjacent to both $v_{1}$ and $v_{2}$. Let $\{0\}$ and $\phi$ be the setlabels of $v_{1}$ and $v_{2}$ respectively. Let $X_{r}$ is the set-label of the vertex $v_{r}$ then set-label of edge $v_{1} v_{r}$ and edge $v_{2} v_{r}$ will be same. It creates the contradiction in bijectiveness of the mapping for edges.

Proposition 3.6. If $f: V(G) \rightarrow 2^{X}$ is a set-harmonious labeling on a graph $G(V, E)$ with non-empty finite set of non-negative integers. Then, every singleton element of $2^{x}$ should be adjacent to the vertex having the set-label either $\{0\}$ or $\phi$.

Proof. Let $G$ be a graph and $f$ be a set-harmonious labeling then the labeling assigned to the edges should be bijective and labeled as the modulo $(|X|)$ of sumset of adjacent vertices. So, all the non-empty sub sets of $X$ should be exactly once assigned to the edges. This is possible only when all singletons are assigned either adjacent with $\{0\}$ or with $\phi$.

Theorem 3.7. A graph $G(V, E)$ have a set-harmonious labeling with the ground set $X$ then, it have $2^{|X|}-1$ number of edges.

Proof. Let $G$ be any set-harmonious graph and $f: V(G) \rightarrow 2^{X}$ is a setharmonious labeling. Then, the associated mapping $f^{*}: E(G) \rightarrow 2^{X}-\phi$ is bijective. So, $|E(G)|=2^{|X|}-1$.

Theorem 3.8. Let $G$ be a set-harmonious labeling with non-empty finite set $X$ of non-negative integers. Then, the cardinality of the ground set $X$ is $\log _{2}[|E(G)|+1]$.

Proof. $G$ be a graph with set-harmonious labeling then by above Theorem 3.7 ,

$$
\begin{aligned}
& |E(G)|=2^{|X|}-1 . \\
\Rightarrow & \log (|E(G)|+1)=\log 2^{X} . \\
\Rightarrow & \log (|E(G)|+1)=X \log 2 . \\
\Rightarrow & \frac{\log (|E(G)|+1)}{\log 2}=X . \\
\Rightarrow & X=\log _{2}[|E(G)|+1] .
\end{aligned}
$$




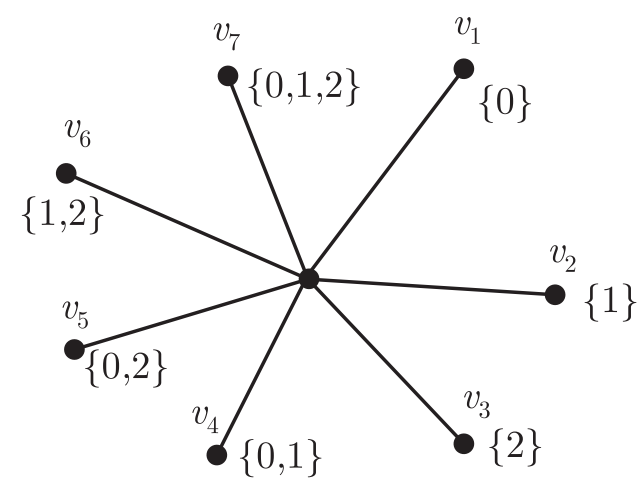

Figure 1

Theorem 3.9. A star graph $S_{1, n}$ have set-harmonious labeling with nonempty finite ground set $X$ if and only if $n=2^{|X|}-1$.

Proof. Let $G=S_{1, n}$ be a star graph with vertices set $\left\{v_{1}, v_{2}, \ldots, v_{n}\right\}$ those are adjacent with the central vertex $v$. Suppose, $G$ have set-harmonious labeling. Then, by above Theorem 3.7, the number of edges in $G=n=2^{|X|}-1$.

Conversely, assume that number of adjacent vertices of $v$ in $S_{1, n}$ are $2^{|X|}-1$ and $X$ is a finite non-empty ground set. We want to prove $S_{1, n}$ have setharmonious labeling. Let $\phi$ be assigned to the central vertex and remaining subsets of $2^{X}$ are assigned to $\left\{v_{1}, v_{2}, \ldots, v_{n}\right\}$ vertices. In this way all the edges of $S_{1, n}$ contains bijective modulo $(|X|)$ sumsets labeling among the vertices.

Hence, $S_{1, n}$ have set-harmonious labeling.

Figure 1 proves the admissibility of set-harmonious labeling for the star graph $S_{1,7}$ for $X=\{0,1,2\}$ where $n=7=2^{|X|}-1$.

Theorem 3.10. A double star graph $S_{m, n}$ have a set-harmonious labeling.

Proof. Let $G=S_{m, n}$ be a double star graph with $|E(G)|=m+n-1$. Let $\left\{v_{1}, v_{2}, \ldots, v_{m}\right\}$ and $\left\{u_{1}, u_{2}, \ldots, u_{n}\right\}$ are two sets of vertices of $S_{m, n}$. Without loss of generality, we can assume that $v_{1}$ and $u_{1}$ are only adjacent vertices in the graph $G$. Let $X$ is the non-empty finite set of non-negative integers. Here, we can label $v_{1}$ as $\phi$ and $u_{1}$ as $\{0\}$ and assign all non-empty sub-sets of $X$ to the remaining vertices. Now, the labeling assign to the edges are modulo $(|X|)$ to the sumset of value assign to the adjacent vertices. Hence, in this way 


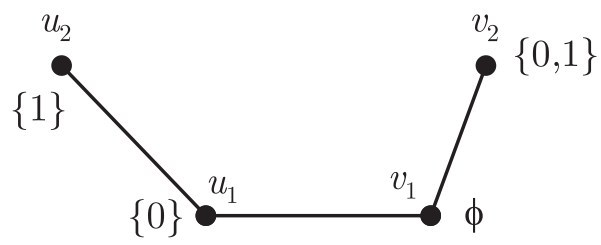

Figure 2

all sub sets of $|X|$ are bijectively assign among the edges. So, $G=S_{m, n}$ is a set-harmonious graph.

Figure 2 proves the admissibility of set-harmonious labeling for the double star graph $S_{2,2}$ for $X=\{0,1\}$ and $|E(G)|=m+n-1=2+2-1=2^{2}-1$.

Theorem 3.11. A complete graph $K_{m}$ does not have a set-harmonious labeling.

Proof. $K_{2}$ is a complete graph with 1 edge. By Theorem 3.7, it does not have set-harmonious labeling. $K_{3}$ is a complete graph with $2^{2}-1=3$ edges but it does not form a bijective mapping along the edges of the graph.

Now, we consider a complete graph with more than three vertices. Assume that the complete graph $K_{m}, m>3$ have a set-harmonious labeling. Then, by Theorem 3.7,

$$
|E(G)|=2^{|X|}-1=\frac{m(m-1)}{2}
$$

where $X$ is a non-empty finite ground set of non-negative integers. Here, $2^{|X|}-1$ is a positive odd integer.

$$
\begin{aligned}
& \Rightarrow \quad \frac{m(m-1)}{2} \text { is also integer. } \\
& \Rightarrow \quad m(m-1) \text { is a multiple of } 2 . \\
& \Rightarrow \quad \text { Either } m=2 k \text { or } m-1=2 k .
\end{aligned}
$$

So,

$$
\begin{aligned}
& 2^{|x|}-1=\frac{2 k(2 k-1)}{2}=k(2 k-1) . \\
\Rightarrow \quad & 2^{|x|}-1=2 k^{2}-k . \\
\Rightarrow \quad & 2 k^{2}-k+1=2^{n} .
\end{aligned}
$$


Hence, $K_{m}$ have a set-harmonious labeling if $2 k^{2}-k+1=2^{n}$ have integral solutions. But, there does not exist any odd positive integer $k$ which satisfies it. Hence, $K_{m}$ does not have a set-harmonious labeling.

Theorem 3.12. $A(m, n)$-Tadpole graph have set-harmonious labeling when $m+n=7$ for $m>2$.

Proof. A $(m, n)$-Tadpole contains a cycle graph $C_{m}$ and a path graph $P_{n}$ with $\left\{v_{1}, v_{2}, \ldots, v_{m}\right\}$ and $\left\{u_{1}, u_{2}, \ldots, u_{n}\right\}$ vertices set respectively. Let $X$ is a non-empty set of non-negative integers. Without loss of generality, we can assume that $C_{m}$ and $P_{n}$ are joined together by a vertex $u_{1}$ and $u_{n}$ is the pendant vertex of the path graph. Here, $m+n=7$. So, the possible cases are as:

1. $m=3, n=4$.

2. $m=4, n=3$.

3. $m=5, n=2$.

We assign $X$ to the pendant vertex of $P_{n}$ and further assign the sub sets of $X$ to the vertices in such a way that each of the edge contain modular sumset of all the vertices over $|X|$ exactly once and makes the bijective mapping corresponding to the edges. Hence, $G$ has a set-harmonious labeling.

Proposition 3.13. Let $G$ be a set-harmonious graph with $p=q+1$ and $X$ is a non-empty finite set of non-negative integers with cardinality $n$. Then subsets of $X$ with cardinality $n-1$ cannot be assigned to the adjacent vertices.

Proof. If $G$ has a set-harmonious labeling and a set-label of cardinality $n-1$ is assigned to $u_{1}$. Then by assigning the sub set of $X$ with cardinality $n-1$ to the adjacent vertices of $u_{1}$, we get the same label corresponding to both edges as $X$. It gives contraction.

Theorem 3.14. No cycle graph $C_{n}$ have set-harmonious labeling.

Proof. Let $C_{n}$ be a cycle graph with $n$ vertices and edges. For being a setharmonious labeling, we must have $2^{|X|}-1$ edges. $C_{n}$ have no pendant vertex. So, by Proposition 3.3, $X$ can not be assigned to any of the vertex. Further, the set labels of cardinality $n-1$ cannot be assigned more than two adjacent vertices because the set labels corresponding to the edges gives $X$. Similarly, nonsingleton sub sets of $X$ with cardinality $n-2$ cannot be assigned to adjacent vertices those have set label as sub set of $X$ with cardinality $n-1$. 
In this way, we require more singletons for assigning to the vertices with the adjacent vertices of set labels of cardinality $n-1, n-2, \ldots$ Hence, $C_{n}$ does not have a set-harmonious labeling.

Theorem 3.15. Every Tree except the path graph have set-harmonious labeling.

Proof. Let $G$ be a tree graph with $|E(G)|=2^{|X|}-1$, where $X$ is a nonempty finite set of nonnegative integers. Then the labeling assign to a node of maximal degree as $\{0\}$ and the node with second maximal degree labeled as $\phi$. The pendant node at highest level in tree labeled as $X$ and labeled the remaining vertices in the tree as mention the results in propositions. Hence, we form a set-harmonious labeled tree.

\section{Conclusion}

In this paper, we have discussed the concept and characterization of set-harmonious graphs and find some graph classes those have set-harmonious labeling. Here, we find the essential requirement of number of edges in the graph for having the set-harmonious labeling. There is also a further scope of research as characterize the different graph classes, verify the existence for different graph operations and establish the necessary and sufficient condition for a graph to admit set harmonious labeling.

\section{References}

[1] B.D. Acharya, Set-valuations and their applications, MRI Lecture notes in Applied Mathematics, The Mehta Research Institute of Mathematics and Mathematical Physics, New delhi, 1983.

[2] B.D. Acharya, K.A. Germina, K.L. Princy and S.B. Rao, Topologically set-graceful graphs, J. Combinat., Inf E System Sci., 27, No. 2-4 (2010), 299-318.

[3] S.W. Golomb, Graph Theory and Computing: How to number a Graph, New-York: Academic Press, 1972.

[4] R.L. Graham and N.J.A. Sloane, On additive bases and harmonious graphs, SIAM Journal on Algebric 83 Discrete Methods, 1, No. 4 (1980), 382-404.

[5] F. Harary, Graph Theory, Addison-Wesley Publishing Company Inc., 1969.

[6] A. Rosa, On certain valuation of the vertices of a graph, in Theory of Graphs, Gordon and Breach, 1967. 
[7] N.K. Sudev and K.A. Germina, A study on integer additive set-graceful graphs, arXiv: 1403.3984 v1[math.Co], 2014.

[8] N.K. Sudev and K.A. Germina, A study on topological integer additive set-labeling of graphs, Electronic Journal of Graph Theory and Applications, 3, No. 1 (2015), 70-84.

[9] U. Thomas and S.C. Mathew, On topological set-indexers of graphs, Advances \& Applications in Discrete Mathematics, 5, No. 2 (2010), 115-130.

[10] D.B. West, Introduction to Graph Theory, Pearson Education Inc., 2001. 
